\title{
A NEW LOOK FOR HAMILTONIAN DYNAMICS
}

\section{by C. W. KILMISTER}

\section{Introduction}

It seems a pity that Hamiltonian dynamics-contact transformations and so on-is regarded as a fearsome subject, too time-consuming to teach to most students; for it is the one branch of dynamics to point a way to new developments in this century. Moreover the basic ideas are extremely simple, but presented in an unfortunate way in all the text-books.

We are interested in a set of transformations of coordinates which leave unchanged the numerical value of a constant two-index tensor $a_{\alpha \beta}(\alpha, \beta=1,2$, $\ldots N)$. That is, the transformations satisfy the differential equation

$$
a_{\alpha \beta}^{\prime}=\frac{\partial x^{\mu}}{\partial x^{\prime \alpha}} \frac{\partial x^{\nu}}{\partial x^{\prime \beta}} a_{\mu \nu}=a_{\alpha \beta}
$$

Clearly (1) splits up into two independent conditions of the same form, one involving the symmetric part of $a_{\alpha \beta}, b_{\alpha \beta}=\frac{1}{2}\left(a_{\alpha \beta}+a_{\beta \alpha}\right)$, and one the antisymmetric part $c_{\alpha \beta}=\frac{1}{2}\left(a_{\alpha \beta}-a_{\beta \alpha}\right)$. The condition involving $b_{\alpha \beta}$ is well-known and easy to deal with. We differentiate with respect to $x^{\prime a}$ and write for a moment

$$
(\mu)=b_{\alpha \beta} \frac{\partial x^{\alpha}}{\partial x^{\prime \mu}} \frac{\partial^{2} x^{\beta}}{\partial x^{\prime \nu} \partial x^{\prime \sigma}} ;
$$

then (1) implies $(\mu)+(v)=0$. By cyclic permutation it follows that $(v)+(\sigma)=0$, $(\sigma)+(\mu)=0$, and so $(\mu)=(v)=0$. It is easy to deduce that the transformation is linear,

where also

$$
x^{\alpha}=C_{\beta}^{\alpha} x^{\prime \beta}+C^{\alpha}
$$

$$
C_{\mu}^{\alpha} b_{\alpha \beta} C_{v}^{\beta}=b_{\mu v} \text {. }
$$

The best-known example of this is obtained by choosing

$$
\begin{aligned}
b_{\alpha \beta}=\delta_{\alpha \beta} & =1 \text { if } \alpha=\beta \\
& =0 \text { otherwise, }
\end{aligned}
$$

so that we have then proved that the only coordinate transformations leaving unchanged the sum of squares are linear ones (and form, actually, the orthogonal group). The remaining condition

$$
c_{\alpha \beta} \frac{\partial x^{\alpha}}{\partial x^{\prime \mu}} \frac{\partial x^{\beta}}{\partial x^{\prime \nu}}=c_{\mu v}
$$

then selects some of these orthogonal transformations as those leaving $c_{\alpha \beta}$ 
also unchanged. This restricts the transformations very severely, but there are two special cases in which the restriction is not so severe:

(i) If $c_{\mu \nu}=0$, so that $a_{\mu \nu}$ is symmetric, we have the whole orthogonal group, the relation between the $x^{\alpha}$ and $x^{\prime \alpha}$ being linear.

(ii) If $b_{\mu \nu}=0$, so that $a_{\mu \nu}$ is antisymmetric, the transformations need not be linear or orthogonal, and this is the case of Hamiltonian dynamics. We shall now limit ourselves to case (ii).

\section{Generators of the Transformation}

The situation for $b_{\mu v}$ is easily described in terms of a quadratic form; for $c_{\mu \nu}$ we can define a bilinear form $c_{\mu \nu} d x^{\mu} d y^{\nu}$ which is obviously unchanged under the transformations. Consider now the integral

$$
I=\int c_{\mu \nu} x^{\mu} d x^{\nu}
$$

For a small variation of the path of integration (and the end-points)

$$
\begin{aligned}
\delta I & =\int c_{\mu \nu}\left(\delta x^{\mu} d x^{\nu}+x^{\mu} d \delta x^{\nu}\right) \\
& =\int c_{\mu v}\left(\delta x^{\mu} d x^{\nu}-d x^{\mu} \delta x^{\nu}\right)+\left[c_{\mu \nu} x^{\mu} \delta x^{\nu}\right],
\end{aligned}
$$

by integration by parts. If $I^{\prime}$ is the corresponding integral with $x^{\prime \mu}$ written for $x^{\mu}$ it now follows from above that

$$
\delta\left(I-I^{\prime}\right)=\left[c_{\mu v}\left(x^{\mu} \delta x^{v}-x^{\prime \mu} \delta x^{\prime v}\right)\right],
$$

which depends only on the end-points and not on the path of integration. Hence there exists a function $W$ of $x^{\mu}, x^{\prime \mu}$ such that

$$
c_{\mu \nu}\left(x^{\mu} \delta x^{\nu}-x^{\prime \mu} \delta x^{\prime \nu}\right)=\delta W\left(x^{\mu}, x^{\prime \mu}\right) .
$$

Further progress is difficult unless we can invert this equation by using the inverse matrix to $c_{\mu v}$. Since $c_{\mu \nu}$ is anti-symmetric the existence of its inverse involves $N$ being even and so we now assume that $N=2 n$. We can then define an inverse $c^{\mu \nu}$, so that $c^{\mu v} c_{v \sigma}=\delta_{\sigma}^{\mu}$, and

$$
x^{\mu}=-c^{\mu \nu} \frac{\partial W}{\partial x^{v}}, \quad x^{\prime \mu}=c^{\mu \nu} \frac{\partial W}{\partial x^{\prime v}}
$$

If, in particular, $W=c_{\mu \nu} x^{\prime \mu} x^{\nu}$ both these conditions are satisfied and moreover $x^{\mu}=x^{\prime \mu}$. This value of $W$ therefore gives the identity transformation. If we take $W=c_{\mu \nu} x^{\prime \mu} x^{\nu}+\delta t K\left(x^{\mu}, x^{\prime \mu}\right)$ where $\delta t$ is small, the small changes in $x^{\mu}$ are given by

$$
\delta x^{\mu}=x^{\prime \mu}-x^{\mu}=\delta t c^{\mu \nu} \frac{\partial K}{\partial x^{\prime \nu}}=\delta t c^{\mu \nu} \frac{\partial K}{\partial x^{\nu}}
$$


to the first order in $\delta t$. If $t$ is some continually evolving parameter this implies that there must exist some function $K=K\left(x^{v}\right)$ such that

$$
\frac{d x^{\mu}}{d t}=c^{\mu \nu} \frac{\partial K}{\partial x^{v}}
$$

\section{The Case of Dynamics}

A dynamical system is specified by a set of generalised coordinates $q_{i}$ $(i=1,2, \ldots, n)$ and a Lagrangian $L=L\left(q^{i}, \dot{q}^{i}\right)$. The corresponding equations of motion are

$$
\dot{p}_{i}=\frac{\partial L}{\partial q^{i}},
$$

where the momenta are defined by

$$
p_{i}=\frac{\partial L}{\partial \dot{q}^{i}}
$$

It follows that

$$
\delta\left(p_{\imath} \dot{q}^{i}-L\right)=\dot{q}^{i} \delta p_{i}-\dot{p}_{i} \delta q^{i},
$$

so that if we use $H$ to denote the result of expressing $p_{i} \dot{q}^{i}-L$ in terms of $p_{i}, q$ only, we have

$$
\dot{q}^{i}=\frac{\partial H}{\partial p_{i}}, \quad \dot{p}_{i}=-\frac{\partial H}{\partial q^{i}} .
$$

These equations can be written in the single form

$$
\dot{x}^{\mu}=\eta^{\mu \nu} \frac{\partial H}{\partial x^{\nu}}
$$

if we define

$$
\begin{aligned}
x^{\mu} & =q^{\mu} & \mu & =1,2, \ldots, n, \\
& =p_{\mu-n} & \mu & =n+1, n+2, \ldots 2 n=N,
\end{aligned}
$$

and

$$
\eta^{i+n}=1, \quad \eta^{i+n i}=-1,
$$

with $\eta^{\mu v}=0$ otherwise. These are the Hamiltonian equations of motion, and they are obviously unchanged under transformations leaving unchanged the anti-symmetric quantity $\eta^{\mu \nu}$ (or, equally, its reciprocal $\eta_{\mu v}$ ). The transformations are contact transformations. The differential equations (2) are equivalent to

$$
\left(q^{i}, q^{\prime j}\right)=0, \quad\left(p_{i}^{\prime}, p_{j}^{\prime}\right)=0, \quad\left(q^{\prime i}, p_{j}^{\prime}\right)=\delta_{j}^{i},
$$

where the Poisson bracket $(\phi, \psi)$ is defined by

$$
(\phi, \psi)=\eta^{\mu \nu} \frac{\partial \phi}{\partial x^{\mu}} \frac{\partial \psi}{\partial x^{\nu}}=\frac{\partial \phi}{\partial q^{i}} \frac{\partial \psi}{\partial p_{i}}-\frac{\partial \phi}{\partial p_{i}} \frac{\partial \psi}{\partial q^{i}}
$$


The bilinear form is now the "bilinear covariant" $d p_{i} d Q^{i}-d q^{i} d P_{i}$, and the condition (3) is that

$$
\left(p_{i} d q^{i}-q^{i} d p_{i}\right)-\left(p_{i}^{\prime} d q^{\prime i}-q^{\prime i} d p_{i}^{\prime}\right)=d W,
$$

but by adding $d\left(p_{i} q^{i}-p_{i}^{\prime} q^{i}\right)$ to both sides this is usually expressed in the form that $p_{i} d q^{i}-p_{i}^{\prime} d q^{\prime i}$ is a complete differential. Moreover the last result of $\S 2$ shows that the Hamiltonian equations of motion themselves describe the unfolding of a contact transformation leaving them invariant.

Most of the results of Hamiltonian dynamics follow very simply in this notation as the reader may now verify for himself by working through one of the standard texts.

KING'S COLLege

LONDON 